\title{
Teoria da Sensibilidade ao Reforçamento: Pressupostos, Procedimentos e Medidas
}

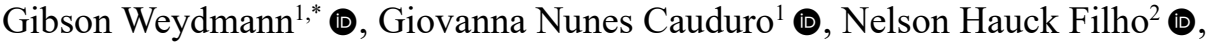 \\ \& Lisiane Bizarro ${ }^{1}$ (D) \\ ${ }^{1}$ Universidade Federal do Rio Grande do Sul, Porto Alegre, RS, Brasil \\ ${ }^{2}$ Universidade São Francisco, Itatiba, SP, Brasil
}

\begin{abstract}
RESUMO - A Teoria da Sensibilidade ao Reforçamento (RST) é um modelo psicobiológico de personalidade derivado de estudos experimentais com humanos e não humanos. Na RST, duas dimensões de personalidade influenciam a reatividade ao ambiente: sensibilidade à recompensa (SR) e sensibilidade à punição (SP). O pressuposto central da teoria é de que há uma relação entre personalidade (SR e SP) e aprendizagem por condicionamento. O objetivo deste artigo é apresentar aos pesquisadores brasileiros a história, as hipóteses centrais e os procedimentos experimentais da RST e discutir as medidas utilizadas para testar os pressupostos da teoria. A RST é um modelo promissor para o estudo da personalidade dentro da psicologia experimental, psiquiatria e neurociências.
\end{abstract}

PALAVRAS-CHAVE: teoria da sensibilidade ao reforçamento, medidas, personalidade, condicionamento

\section{Reinforcement Sensitivity Theory:Assumptions, Procedures, and Measures}

\begin{abstract}
The Reinforcement Sensitivity Theory (RST) is a biological model of personality derived from experimental studies with humans and nonhumans. In RST, two personality dimensions influence reactivity to the environment: sensitivity to reward (SR) and sensitivity to punishment (SP). The central assumption of the theory is that there is a relationship between personality (SR and SP) and conditioning learning. This article aims to introduce Brazilian researchers to the history, central hypotheses, and experimental procedures of RST and discuss the measures used to test the theoretical assumptions. RST is a promising model for the study of personality within experimental psychology, psychiatry, and neuroscience.
\end{abstract}

KEYWORDS: reinforcement sensitivity theory, measures, personality, conditioning

Modelos biológicos de personalidade foram construídos com o objetivo de explicar a estabilidade ao longo do tempo de padrões comportamentais (i.e., traços psicológicos) por meio de mecanismos que passaram por seleção natural na história da espécie. Tais modelos possibilitam o retorno da ênfase aos processos psicológicos básicos (e.g., aprendizagem, atenção e motivação) para a criação de medidas psicológicas, o que pode ajudar a preencher a lacuna empírica entre personalidade e psicopatologia (Bijttebier et al., 2009). Diversas teorias psicobiológicas foram elaboradas com o intuito de explicar a estabilidade de traços psicológicos, sendo uma das mais citadas a Teoria da Sensibilidade ao Reforçamento (do inglês, Reinforcement
Sensitivity Theory - RST) (Corr, 2016; Gray \& McNaughton, 2000).

Com mais de 40 anos de pesquisa, o pressuposto principal da RST é que traços de personalidade associados à sensibilidade a estímulos ambientais influenciam a aprendizagem por reforçamento (Ávila \& Torrubia, 2008; Gray \& McNaughton, 2000; Leue \& Beauducel, 2008). Diversos questionários de autorrelato e procedimentos de condicionamento foram elaborados com o intuito de testar a relação entre personalidade e condicionamento estabelecida pela RST (Corr, 2004, 2016; Leue \& Beauducel, 2008). Embora o modelo de personalidade da RST esteja em pleno desenvolvimento, não foram encontradas referências

\footnotetext{
*E-mail: gibsonjw6@gmail.com

- Submetido: 13/02/2019; Revisado: 07/05/2020; Aceito: 15/06/2020.
} 
em língua portuguesa sobre a teoria, suas hipóteses e instrumentos.

Dessa forma, o presente estudo visa apresentar os pressupostos principais da RST e alguns dos procedimentos necessários para o teste da relação entre personalidade e reforçamento estabelecidos por estudos da área. Em seguida, as principais medidas psicométricas de autorrelato e tarefas operantes que podem ser utilizadas para testar a relação entre personalidade e condicionamento proposta pela RST são apresentadas.

\section{BREVE HISTÓRICO DA RST}

Diferente de outros modelos biológicos de personalidade, a RST foi desenvolvida com base em estudos experimentais e farmacológicos com modelos animais (Walker et al., 2017). O processo de criação da teoria e estabelecimento dos principais constructos surgiu a partir das críticas de Jeffrey A. Gray sobre o modelo extroversão-neuroticismo de Hans Eysenck (Gray, 1982), que por sua vez também propunha uma teoria psicobiológica da personalidade.

A teoria de Eysenck possuía dois traços de personalidade em seu início, extroversão e neuroticismo, com níveis baixos destes traços representando, respectivamente, introversão e estabilidade emocional (Pickering \& Corr, 2008). Gray foi aluno de Eysenck e, sob indicações de seu tutor, dedicou-se a traduzir obras de psicólogos e fisiologistas russos, como Pavlov e Teplov. O contato com a proposta de Pavlov influenciou tutor e aluno a refletirem sobre a natureza da personalidade, não como padrões comportamentais, mas sim como manifestações distintas do sistema nervoso e de mecanismos básicos de aprendizagem (Pickering \& Corr, 2008). Influenciado por esta ideia, Eysenck propôs, após diversas revisões de sua teoria, que a associação observada entre introversão, neuroticismo e neuroses clínicas estava relacionada à uma facilitação na aquisição de respostas de medo e ansiedade em processos de condicionamento clássico (Eysenck, 1979). Dito de outra maneira, Eysenck suponha que indivíduos introvertidos e neuróticos aprenderiam com menos tentativas e maior reatividade do sistema nervoso a relação entre um estímulo condicionado (e.g., elevador) e um estímulo incondicionado aversivo (e.g., isolamento após o elevador quebrar entre andares).

Acrítica de Gray (1982) ao modelo de Eysenck era dirigida à proposta do último de que uma personalidade introvertida sempre facilitaria o processo de condicionamento. Em um estudo realizado pelo próprio Eysenck (Eysenck \& Levey, 1972), esta hipótese foi falseada. Sujeitos introvertidos e extrovertidos, distribuídos em grupos pelos escores obtidos em um instrumento de personalidade, foram avaliados com relação à velocidade da aquisição de respostas condicionadas de piscar. No experimento, um som (estímulo condicionado) era apresentado randomicamente em conjunto com um jato de ar (estímulo incondicionado) no olho dos participantes. Ao contrário do esperado por Eysenck, frequências maiores da resposta de piscar diante do estímulo sonoro ocorreram apenas em indivíduos com níveis maiores de impulsividade, um subfator de extroversão, e neuroticismo, oposto à introversão (Eysenck \& Levey, 1972).
Amplamente baseado em estudos experimentais da neurologia e da farmacologia, Gray (1982) deduziu que a relação entre personalidade e condicionamento poderia ser mais bem explicada pelo entendimento de como áreas distintas do sistema nervoso mediavam a aprendizagem em experimentos de condicionamento. Na proposta de Gray, dois fatores de reatividade ao ambiente extraídos teoricamente do modelo extroversão-neuroticismo poderiam influenciar o condicionamento: a Sensibilidade à Recompensa ${ }^{1}$ (SR), um fator próximo da extroversão e com características do neuroticismo; e a Sensibilidade à Punição (SP), um fator próximo do neuroticismo e com componentes da introversão (Corr, 2004; Gray \& McNaughton, 2000). A proposta de Gray (1982) era de que os fatores SR e SP teriam uma relação causal com os constructos de extroversão e neuroticismo.

Cada traço de personalidade na teoria de Gray foi pensado com base na ação de sistemas cerebrais. A SR seria regulada pela reatividade do Sistema de Aproximação Comportamental (Behavioral Approach System-BAS), um conjunto neuroanatômico sensível a estímulos apetitivos (i.e., que geram aproximação comportamental) condicionados, relacionado a afetos positivos e ao traço de impulsividade. Já a SP foi dividida em dois sistemas: Sistema de Luta-Fuga (Fight-Flight System-FFS), sensível a estímulos aversivos incondicionados e relacionado às emoções de medo e pânico; e Sistema de Inibição Comportamental (Behavioral Inhibition System-BIS), sensível a estímulos aversivos condicionados, mudanças extremas em contingências e relacionado ao traço de ansiedade (Pickering \& Corr, 2008; Gray, 1982). Nessa primeira versão da RST, os três sistemas apresentados acima eram considerados independentes, portanto, as dimensões de SR e SP influenciavam processos de aprendizagem e reatividade a estímulos de maneira separada (Corr, 2004).

Por ser uma teoria derivada de dados, a RST passou por uma revisão anos mais tarde (Gray \& McNaughton, 2000), devido principalmente ao avanço em duas áreas de pesquisa: a etofarmacologia e a neurociência comportamental, cujos dados auxiliavam os pesquisadores a compreender de que

1 A teoria de Gray foi criada com base na literatura pavloviana sobre a condicionabilidade do sistema nervoso, o que a afasta dos estudos de Skinner e da Análise do Comportamento. Por fazer parte das neurociências, a RST utiliza o termo reforçamento de maneira ampla, referindo-se ao aumento na força da relação estímulo-resposta ou resposta-consequência. Termos como "recompensa" são utilizados para se referir a estímulos incondicionados (e.g., comida) ou condicionados, utilizados como reforçadores em tarefas operantes. 
maneira arranjos ambientais modulavam reações básicas de defesa dos organismos (McNaughton \& Corr, 2008). Gray e McNaughton (2000) identificaram uma diferença entre respostas de medo e ansiedade relevante para o teste de hipóteses da RST, segundo a qual respostas de fuga e esquiva pareciam ocorrer diante de estímulos aversivos enquanto respostas de esquiva passiva e ansiedade seriam decorrentes de um conflito entre se aproximar ou não de um ambiente potencialmente perigoso. A distância do estímulo aversivo é importante para diferenciar medo e ansiedade, como exemplifica o estudo de neuroimagem de Mobbs e colegas (2007) com seres humanos, que mostrou que a proximidade do estímulo aversivo em uma medida virtual de luta ou fuga ativa com mais intensidade a substância cinzenta periaquedutal, área relacionada ao medo. Diante dos novos dados acerca da diferença entre medo e ansiedade, Gray e McNaughton (2000) publicaram a versão revisada da RST que começou a ser reconhecida como revised Reinforcement Sensitivity Theory (rRST).

A partir da rRST, o traço de personalidade SP passou a ser dividido em BIS e Sistema de Luta-Fuga-Congelamento (do inglês, Fight-Flight-Freeze System-FFFS), sistemas responsáveis pelas respostas de ansiedade e medo, respectivamente (Gray \& McNaughton, 2000). O FFFS substituiu o antigo sistema $F F S$ e, na rRST, ele reage a estímulos aversivos condicionados e incondicionados que estão presentes no ambiente, possibilita a sensação subjetiva de medo e facilita a aprendizagem de respostas de esquiva como a luta, a fuga e o congelamento (Gray \& McNaughton, 2000). O FFFS está vinculado principalmente ao funcionamento da substância cinzenta periaquedutal e amígdala (Gray \& McNaughton, 2000; McNaughton \& Corr, 2008). O funcionamento do BIS, por sua vez, passou a ser associado a situações de conflito entre BAS e FFFS (Gray \& McNaughton, 2000). Situações de conflito ocorrem quando uma resposta que produz um reforçador elimina a ocorrência de outro com magnitude semelhante ou quando as consequências das respostas comportamentais são incertas, como ocorre em medidas de tomada de decisão por tentativa e erro. A ansiedade produzida pelo BIS é eliciada em conjunto com respostas de esquiva passiva e aproximação defensiva durante o conflito pela via septohipocampal do cérebro. A esquiva passiva é caracterizada pela supressão de respostas comportamentais que estavam em andamento quando há chances de punição, enquanto a aproximação defensiva é uma exploração cautelosa e lenta do ambiente, durante a qual recursos cognitivos como a atenção e a memória são utilizados para identificar perigo (McNaughton \& Corr, 2008).

$\mathrm{Na}$ rRST, inferências sobre o funcionamento neuroquímico dos sistemas cerebrais foram realizadas e os sistemas BIS e FFFS foram atribuídos a vias serotoninérgicas do cérebro (McNaughton \& Corr, 2008). Anos mais tarde, o alelo menor do gene 5-HTTLPR, ligado a sintomas de depressão e ansiedade e uma maior ação da serotonina em áreas subcorticais ligadas ao medo e ansiedade, foi associado a maiores escores de SP avaliado por autorrelato (Whisman et al., 2011).

Na versão nova da teoria, o sistema BAS reage diante de estímulos apetitivos condicionados e incondicionados e possui um funcionamento ligado à via mesocorticolímbica de dopamina (Gray \& McNaughton, 2000; Pickering \& Corr, 2008). Estudos genéticos com polimorfismos ligados à disponibilidade de dopamina e de receptores dopaminérgicos (e.g., DRD2 e COMT) indicam que, de fato, a SR (BAS) avaliada por autorrelato é maior em pessoas com interações genéticas ligadas à uma maior disponibilidade de dopamina na via mesocorticolímbica (Reuter et al., 2006). Isto ocorre mesmo com fatores de personalidade associados à SR, como a busca por sensações, que é maior em adultos e familiares de primeiro grau com o alelo maior do gene DRD4 (Benjamin et al., 1996).

\section{PRESSUPOSTOS E PROCEDIMENTOS}

O principal pressuposto da RST é que traços de personalidade influenciam a aprendizagem por condicionamento e a hipótese derivada da teoria é que tal efeito é decorrente da reatividade dos sistemas cerebrais BAS, BIS e FFFS (Gray, 1982; Gray \& McNaughton, 2000). Assim, na primeira elaboração da RST havia a suposição de que altos níveis de SR facilitariam a aprendizagem por reforço positivo e a aquisição de respostas emocionais positivas por condicionamento clássico, enquanto altos níveis de SP facilitariam a aprendizagem de respostas de esquiva e a aquisição de reações emocionais aversivas por condicionamento clássico (Gray, 1982).

Assim como ocorre com outras teorias da personalidade, a avaliação dos traços de personalidade da RST é realizada majoritariamente por meio de instrumentos de autorrelato com fatores de SR e SP (Corr, 2016). No entanto, diversos pesquisadores defendem que nos estudos de validação desses instrumentos, os fatores de SR e SP devem estar relacionadas a medidas de condicionamento (Ávila \& Torrubia, 2008; Corr, 2004; Leue \& Beauducel, 2008; Markett et al., 2014). A correspondência dos instrumentos de autorrelato com dados experimentais é importante porque Gray (1982) atrelou sua teoria de personalidade aos resultados de experimentos de condicionamento; sem esses resultados, os instrumentos de autorrelato não estariam de acordo com a RST.

Há, portanto, procedimentos necessários para o teste da relação entre personalidade e condicionamento da RST/rRST (Corr, 2004; Leue \& Beauducel, 2008). Por não haver ainda um experimento ideal para diferenciar SR e SP, a personalidade dos participantes deve ser 
avaliada por questionários. Os escores obtidos podem, então, ser relacionados com dados comportamentais de diferentes formas: correlacionando desfechos de medidas de condicionamento com os dados de personalidade (Corr \& Thompson, 2014; Smillie et al., 2007), comparando grupos contrastantes nos escores de personalidade (e.g., $\mathrm{SR}+\mathrm{e} \mathrm{SR}-$ ) com relação à performance na aprendizagem de respostas condicionadas ou operantes (Ávila \& Parcet, 2000; Kambouroupoulos \& Staiger, 2004), ou agrupando os participantes a partir do desempenho nas medidas de reforçamento e comparando estes grupos com relação aos dados de personalidade (Aberg et al., 2016).

Estudos realizados antes da elaboração de medidas de autorrelato da RST com experimentos de condicionamento clássico vão ao encontro do pressuposto central da teoria. $\mathrm{Na}$ maioria desses estudos, os sujeitos de pesquisa eram agrupados pelas características de personalidade do modelo de Eysenck a priori, criando assim grupos contrastantes de extroversão $(\mathrm{E}+/ \mathrm{E}-)$ e neuroticismo $(\mathrm{N}+/ \mathrm{N}-)$ (Corr, 2004). Dois procedimentos de condicionamento clássico frequentemente utilizados para avaliar a aquisição de respostas condicionadas eram o condicionamento do reflexo de piscar os olhos, mencionado no estudo de Eysenck e Levey (1972), e o condicionamento de respostas galvânicas da pele (Corr, 2004). As respostas galvânicas da pele são reações do sistema nervoso simpático que podem ser medidas por eletromiografia. Corr (2004) relata alguns experimentos que mostram que estímulos neutros associados a estímulos incondicionados apetitivos (e.g., imagens eróticas) eliciam mais rapidamente respostas galvânicas em pessoas com E+/ $\mathrm{N}-$, enquanto que estímulos neutros associados a estímulos incondicionados aversivos (e.g., jato de ar nos olhos) eliciam mais rapidamente respostas de piscar em pessoas com E-/ $\mathrm{N}+$ (Corr, 2004).

Resultado semelhantes foram encontrados em experimentos com o condicionamento operante. A meta análise realizada por Leue e Beauducel (2008) revela uma série de estudos que vão ao encontro das hipóteses da RST. Em diversos procedimentos operantes, indivíduos $\mathrm{E}+$ / $\mathrm{N}$ - demonstraram uma frequência maior de respostas em condições de reforço positivo, enquanto indivíduos $\mathrm{E}-$ $\mathrm{N}+$ demonstraram uma maior frequência de respostas de esquiva em condições de reforço negativo. Leue e Beauducel também observaram que grupos $\mathrm{E}+\mathrm{N}-$ demonstram um tempo de reação médio menor diante de estímulos previamente correlacionados com reforçadores positivos, enquanto grupos $\mathrm{E}-/ \mathrm{N}+$ tendem a demonstrar um tempo de reação médio mais lento frente a estímulos correlacionados com punição. Ambos os resultados são importantes, porque enquanto a frequência das respostas operantes indica o efeito das consequências sobre a resposta, a média do tempo de reação em milissegundos para cada tentativa indica a relação aprendida entre estímulo antecedente e consequência ao longo do procedimento operante (Leue \& Beauducel, 2008). Apesar de não utilizarem medidas específicas de SR ou SP, os estudos mencionados acima vão ao encontro da hipótese de Gray (1982), visto que a relação $\mathrm{E}+\mathrm{N}-\mathrm{e} \mathrm{E}-/ \mathrm{N}+$ serviu de base, respectivamente, para a elaboração das dimensões de SR e SP.

As revisões de Ávila e Torrubia (2008), Corr (2004) e Leue e Beauducel (2008) indicam que a maior parte dos estudos sobre RST e condicionamento foram realizados tendo como base a versão antiga da RST, por isso não incluíam medidas de conflito importantes para distinguir respostas ligadas ao sistema BIS da rRST. Além disso, a ausência de medidas de autorrelato específicas da RST prejudicava a interpretação dos dados, ainda que estivessem de acordo com as propostas de Gray sobre o modelo de Eysenck (Gray, 1982).

Com a criação da rRST, Gray \& McNaughton (2000) acrescentaram novas hipóteses sobre como a SP está ligada à aprendizagem por condicionamento, o que tornou complexo o teste de hipóteses da teoria. Um exemplo é a mudança dos sistemas FFFS e BIS da rRST, que passaram a se relacionar com a resposta de esquiva ativa no primeiro caso e aproximação defensiva no segundo, e que antes (i.e., na RST) eram relevantes para as respostas de fuga diante de estímulos incondicionados e condicionados, respectivamente (McNaughton \& Corr, 2008).

Outra mudança decorrente da revisão da teoria está relacionada à suposição de que SR e SP influenciam a aprendizagem por condicionamento de maneira independente. A hipótese dos sistemas separados (HSS) postula que os sistemas BAS, BIS e FFFS são ortogonais, influenciando a reatividade a estímulos apetitivos, a conflitos e a estímulos aversivos de maneira independente (Corr, 2004). Na HSS, o traço SR (BAS) não influencia, por exemplo, a aprendizagem por esquiva que é atribuída a maiores níveis de SP.

A partir de uma ampla revisão dos estudos experimentais da RST, Corr (2004) apresentou evidências de que as dimensões de SR (BAS) e SP (BIS e FFFS) podem funcionar em conjunto. A hipótese dos sistemas conjuntos (HSC) postula que BAS, BIS e FFFS podem apresentar um efeito antagonista ou facilitatório entre si. A HSC tem por base dados que indicam que, comparados com pessoas que reportam $\mathrm{SR}+/ \mathrm{SP}+$, indivíduos com $\mathrm{SR}-/ \mathrm{SP}+$ tendem a apresentar um maior reflexo de sobressalto diante de estímulos aversivos e maiores níveis de afeto negativo (Corr, 2004). Indivíduos com SR+/SP-, por outro lado, apresentam maior reatividade a estímulos apetitivos e maior impulsividade em medidas de controle inibitório, que avaliam a capacidade de inibir respostas diante de sinais stop (Corr, 2004). Indo ao encontro da hipótese de Corr (2004), em experimentos nos quais uma mesma resposta é associada tanto à punição quanto ao reforço (i.e., tarefas de conflito), efeitos de acordo com a HSC ocorrem devido à ativação conjunta dos sistemas, com participantes SR-/ $\mathrm{SP}+$ apresentando uma maior lentidão no tempo de reação e uma frequência menor de respostas diante de estímulos que sinalizam punição (Leue \& Beauducel, 2008). 
Com a mudança da RST para a rRST, medidas de condicionamento clássico (e.g., reações galvânicas na pele) e operante (e.g., frequência de respostas) previamente utilizados continuaram sendo relevantes para o teste da relação entre personalidade e condicionamento. Entretanto, diante dos resultados comportamentais obtidos nos testes da rRST, medidas adicionais foram adotadas, tais como erros de omissão; isto é, a não emissão de uma resposta natural na presença de estímulos antecedentes que sinalizam punição. As respostas de aproximação defensiva e esquiva passiva do sistema BIS são representadas tanto pelos erros de omissão quanto pelos tempos de reação mais lentos diante de estímulos que sinalizam punição (Leue \& Beauducel. 2008). Antes de discutir as medidas de condicionamento, no entanto, é necessário analisar as principais medidas psicométricas de autorrelato utilizadas para avaliar os traços SR e SP.

\section{MEDIDAS PSICOMÉTRICAS DE AUTORRELATO}

Medidas de autorrelato de SR e SP são elaboradas desde 1989 e algumas delas utilizam os nomes dos sistemas neuroanatômicos (BAS, BIS e FFFS) como título das escalas e subescalas $^{2}$ (Corr, 2016). Os maiores desafios encontrados na elaboração de medidas psicométricas de autorrelato estão relacionados à multidimensionalidade do sistema BAS e à distinção necessária entre BIS e FFFS (Corr, 2016; Krupić et al., 2016). A avaliação do fator SR por meio de autorrelato é mais adequadamente representada por múltiplos fatores, dado que reações de impulsividade, afetos positivos e busca ativa por reforçadores costumam ser associados ao sistema BAS (Carver \& White, 1994; Corr \& Cooper, 2016; Krupić et al., 2016). Na avaliação do fator SP, alguns problemas são encontrados nos instrumentos de autorrelato elaborados com base na versão antiga da RST, que não incluem a diferenciação entre BIS e FFFS, a qual é particularmente importante para diferenciar psicopatologias como fobias e transtornos de ansiedade (Bijttebier et al., 2009; Gray \& McNaughton, 2000). Já instrumentos elaborados após a rRST costumam apresentar fragilidades como correlações muito altas (acima de 0,70 ) ou muito baixas entre (abaixo de 0,10 ) entre subescalas de BIS e FFFS, sendo que associações moderadas (entre 0,40 e 0,60) são esperadas dada a correspondência desses sistemas com o fator de SP (Krupić et al., 2016). Para auxiliar na escolha das medidas psicométricas da rRST, algumas considerações acerca dos instrumentos mais utilizados para avaliar RST/rRST foram dispostas na Tabela 1 .

O Behavioral Inhibition System/Behavioral Approach System (BIS/BAS; Carver \& White, 1994) foi uma das primeiras escalas construídas para avaliar a RST. O instrumento contém duas subescalas, uma para avaliar BIS/SP (sete itens) e a segunda para investigar o fator multidimensional BAS/SR (13 itens divididos entre Drive, Reward Responsiveness, e Fun Seeking). Devido à sua ampla utilização, a BIS/BAS tem sua aplicabilidade e propriedades psicométricas testadas frequentemente (Corr, 2016). É possível destacar duas principais limitações do

2 Os nomes dos instrumentos foram mantidos no idioma original pois entende-se que a tradução desses deve ocorrer apenas por meio do processo de adaptação transcultural, de modo a manter a coerência e adequação entre o instrumento original e o instrumento traduzido. instrumento: (1) a multidimensionalidade da subescala BAS foi identificada a partir de análises fatoriais exploratórias e, apesar de estar de acordo com a teoria, não era esperada pelos criadores do instrumento; e (2) a ausência de diferenciação fatorial das subescalas BIS e FFFS, uma vez que ambas são avaliadas pelo fator BIS.

O Sensitivity to Punishment and Sensitivity to Reward Questionnaire (SPSRQ) foi elaborado a partir da RST, mas é utilizado em diversos estudos mesmo após a proposição da rRST. Por ter sido elaborado com base na premissa de que os fatores SR e SP são unidimensionais, o uso do SPSRQ compromete a avaliação dos fatores subjacentes a SR e SP e seu uso deve ser desestimulado. O instrumento é dividido em duas subescalas: uma para avaliar SR (24 itens) e outra para avaliar SP (24 itens). Dados psicométricos da escala são descritos por Torrubia et al. (2001). Uma versão revisada da escala foi publicada por Conner et al. (2018) com a proposta de melhorar o entendimento dos itens da versão original e propor um modelo Likert de cinco pontos como alternativa de escala de resposta. A principal limitação da SPSRQ está na unidimensionalidade das subescalas SR e SP, o que diminui a sensibilidade do questionário à pluralidade de fatores da teoria.

$\mathrm{Na}$ tentativa de operacionalizar as mudanças teóricas da rRST, Jackson (2009) propôs a construção do Jackson-5, um instrumento cujo principal objetivo era aferir adequadamente os comportamentos esperados para os diferentes sistemas da teoria revisada. O principal diferencial da escala é a proposição de cinco subescalas, por meio das quais o autor visa avaliar BAS, BIS e os três fatores de FFFS separadamente (Jackson, 2009). Apesar de apresentar características psicométricas consideradas adequadas (descritos por Jackson, 2009), a unidimensionalidade de BAS negligencia a literatura da teoria, que reforça a pluralidade de variáveis latentes do sistema SR (Krupíc et al., 2016). Outra limitação importante está nas correlações entre BIS e os fatores de FFFS, cujos valores são próximos de zero.

Cinco anos após a apresentação da escala Jackson-5, Smederevac et al. (2014) publicaram os primeiros estudos acerca da criação e validação do Reinforcement Sensitivity Questionnaire (RSQ). O instrumento foi elaborado para avaliar a rRST a partir de cinco fatores: BAS, BIS, Fight, 
Tabela 1

Instrumentos Usados para Avaliar RST e rRST

\begin{tabular}{|c|c|c|c|c|c|c|c|}
\hline \multirow{2}{*}{ Instrumento } & \multirow{2}{*}{$\begin{array}{l}\mathbf{N}^{0} \text { de } \\
\text { itens }\end{array}$} & \multirow{2}{*}{$\begin{array}{c}\text { Tipo de } \\
\text { escala de } \\
\text { resposta }\end{array}$} & \multicolumn{3}{|c|}{ Número de fatores por sistema } & \multirow{2}{*}{ Potencialidades } & \multirow{2}{*}{ Limitações } \\
\hline & & & BAS & BIS & FFFS & & \\
\hline $\mathrm{BIS} / \mathrm{BAS}$ & 20 & $\begin{array}{l}\text { Likert de } \\
\text { quatro } \\
\text { pontos }\end{array}$ & $\begin{array}{l}3 \text { (Drive, Reward } \\
\text { Responsiveness, e } \\
\text { Fun Seeking) }\end{array}$ & 1 & 0 & $\begin{array}{l}\text { - Traduzida para } \\
\text { diversos idiomas } \\
\text { - Escala breve } \\
\text { - Propriedades } \\
\text { psicométricas robustas }\end{array}$ & $\begin{array}{l}\text { - Não diferencia } \\
\text { BIS e FFFS } \\
\text { - Sem justificativa } \\
\text { teórica para divisão } \\
\text { da subescala BAS }\end{array}$ \\
\hline SPSRQ & 48 & Sim ou não & 1 & 1 & 0 & $\begin{array}{l}\text { - Bons índices de } \\
\text { confiabilidade } \\
\text { - Boas correlações entre } \\
\text { itens nos fatores }\end{array}$ & $\begin{array}{l}\text { - Não diferencia } \\
\text { BIS e FFFS } \\
\text { - SR unidimensional }\end{array}$ \\
\hline Jackson-5 & 30 & $\begin{array}{l}\text { Likert } \\
\text { de cinco } \\
\text { pontos }\end{array}$ & 1 & 1 & $\begin{array}{c}3 \text { (Fight, Flight, } \\
\text { Freeze) }\end{array}$ & $\begin{array}{l}\text { - Alinhamento } \\
\text { teórico devido à alta } \\
\text { correlação entre BAS e } \\
\text { Extroversão } \\
\text { - Divide BIS e FFFS }\end{array}$ & $\begin{array}{l}\text { - Correlações entre } \\
\text { BIS e subfatores } \\
\text { de FFFS próximas } \\
\text { de zero } \\
\text { - SR } \\
\text { unidimensional, } \\
\text { poucos itens }\end{array}$ \\
\hline RSQ & 29 & $\begin{array}{l}\text { Likert de } \\
\text { quatro } \\
\text { pontos }\end{array}$ & 1 & 1 & $\begin{array}{c}3 \text { (Fight, Flight, } \\
\text { Freeze) }\end{array}$ & $\begin{array}{l}\text { - Desenvolvido a partir } \\
\text { dos pressupostos da } \\
\text { rRST } \\
\text { - Boa coerência } \\
\text { teórica (bons índices } \\
\text { no modelo de análise } \\
\text { fatorial confirmatória) }\end{array}$ & $\begin{array}{l}\text { - BAS } \\
\text { unidimensional } \\
\text { - Altas correlações } \\
\text { entre BIS e Flight e } \\
\text { BIS e Freeze }\end{array}$ \\
\hline rRST-Q & 31 & $\begin{array}{l}\text { Likert de } \\
\text { quatro } \\
\text { pontos }\end{array}$ & 1 & 1 & $1+$ Fight & $\begin{array}{l}\text { - Distinção entre os } \\
\text { itens de BAS em } \\
\text { diferentes facetas } \\
\text { - Bons índices de } \\
\text { consistência interna dos } \\
\text { fatores }\end{array}$ & $\begin{array}{l}\text { - BAS } \\
\text { unidimensional }\end{array}$ \\
\hline RST-PQ & 65 & $\begin{array}{l}\text { Likert de } \\
\text { quatro } \\
\text { pontos }\end{array}$ & $\begin{array}{l}4 \text { (Reward Interest, } \\
\text { Goal-Drive } \\
\text { Persistence, } \\
\text { Reward Reactivity, } \\
\text { Impulsivity) }\end{array}$ & 1 & 1 & $\begin{array}{l}\text { - Desenvolvida a partir } \\
\text { de modelos animais } \\
\text { - Alta adequação } \\
\text { teórica e empírica } \\
\text { - Escala separada para } \\
\text { avaliar Defensive Fight }\end{array}$ & $\begin{array}{l}\text { - Amostra pequena } \\
\text { no estudo de } \\
\text { validação; }\end{array}$ \\
\hline RSQ-17 & 17 & $\begin{array}{l}\text { Likert de } \\
\text { quatro } \\
\text { pontos }\end{array}$ & 1 & 1 & 3 & $\begin{array}{l}\text { - Versão reduzida da } \\
\text { RSQ-29 } \\
\text { - Mantidos os itens com } \\
\text { maior adequação ao } \\
\text { modelo } \\
\text { - Maior distinção entre } \\
\text { os itens e fatores de } \\
\text { BIS e FFFS }\end{array}$ & $\begin{array}{l}\text { - BAS } \\
\text { unidimensional }\end{array}$ \\
\hline
\end{tabular}

Nota. BIS/BAS = Behavioral Inhibition System/Behavioral Activation System $;$ SPSRQ = Sensitivity to Punishment and Sensitivity to Reward Questionnaire; $R S Q=$ Reinforcement Sensitivity Questionnaire; $r R S T-Q=$ Revised Reinforcement Sensitivity Theory Questionnaire; $R S T$ - $P Q=$ Reinforcement Sensitivity Theory of Personality Questionnaire; RSQ-17 = Reinforcement Sensitivity Questionnaire - Versão Reduzida; BAS = Behavioral Approach System; BIS = Behavioral Inhibition System; FFFS = Fight-Flight-Freeze System; SR = Sensibilidade à Recompensa; $S P=$ Sensibilidade à Punição.

Flight e Freeze. Apesar da preocupação dos autores em fornecer uma medida adequada à rRST, foram encontradas fragilidades nas propriedades psicométricas da RSQ, como as associações positivas e negativas muito altas, em vez de moderadas, entre BIS e os subfatores de FFFS (e.g., Flight, $r$ $=.73 ;$ Freeze, $r=-.86$ ). Outro aspecto da medida que diverge da literatura é a presença de apenas um fator para avaliar BAS (Corr, 2016). Recentemente, Čolović et al. (2018) disponibilizaram uma versão reduzida do RSQ (Smederevac et al., 2014), com 17 itens (RSQ-17). Entretanto, quando comparados à versão completa da escala, a versão reduzida apresentou valores menores de consistência interna, indicando maior imprecisão do instrumento (Čolović et al., 2018).

Diferentemente dos demais instrumentos propostos após a rRST, o Revised Reinforcement Sensitivity Theory Questionnaire (rRST-Q) teve o objetivo principal de diferenciar e avaliar os sistemas BIS e FFFS adequadamente. 
A escala é composta por três fatores: BAS, BIS e FFFS. A versão original do instrumento foi elaborada em alemão e posteriormente traduzida e adaptada para o inglês (Reuter et al., 2015). Apesar dos cuidados adotados pelos autores na construção do instrumento, as distinções entre BIS e FFFS são frágeis. Além disso, apesar da subescala BAS incluir itens com distinções comportamentais importantes (e.g., entre impulsividade e esforço), ela é avaliada como unifatorial (Reuter et al., 2015).

Corr e Cooper (2016) propuseram o Reinforcement Sensitivity Theory of Personality Questionnaire (RST-PQ), cujo processo de criação englobou critérios adicionais a fim de minimizar os problemas encontrados em instrumentos anteriores. A principal diferença na elaboração do questionário foi a inclusão de respostas observadas em modelos animais diante de situações de aproximação defensiva, associadas ao BIS. O RST-PQ aparenta maior sensibilidade à teoria ao apresentar seis fatores, sendo quatro associados ao BAS, um ao BIS, um ao FFFS e uma escala adicional de Defensive Fight. A reatividade do BAS é avaliada por meio das subescalas Reward Interest, GoalDrive Persistence, Reward Reactivity e Impulsivity. O fator BIS é composto por itens que refletem diferentes respostas de ansiedade e um fator FFFS para avaliar respostas associadas ao medo. A escala de Defensive Fight é considerada à parte para avaliar respostas de luta diante de estímulos aversivos (Corr \& Cooper, 2016).

$\mathrm{O}$ tratamento dado à BAS, por meio da diferenciação entre os quatro fatores, pode ser considerado um ponto forte da escala, uma vez que representa o embasamento teórico adequado. Os cuidados adotados pelos autores parecem refletir na maior adequação da medida, uma vez que os resultados encontrados representam adequadamente os pressupostos teóricos da rRST e possuem boas propriedades psicométricas (apresentadas com maior detalhamento em Corr \& Cooper, 2015).

\section{Recomendações para a escolha de medidas psicométricas}

As primeiras escalas apresentadas após a rRST serviram principalmente para tentar operacionalizar a diferenciação adequada de BIS e FFFS, mas apresentaram fragilidades em suas estruturas psicométricas. Além de correlações moderadas a altas entre BIS e FFFS, observadas nos resultados do RSQ (Smederevac et al., 2014) e do rRST-Q (Reuter et al., 2015), a presença de apenas um fator para avaliar BAS na maior parte dos instrumentos também pode ser considerada uma importante limitação das medidas disponíveis (Jackson, 2009; Smederevac et al., 2014; Reuter et al., 2015). Cabe destacar que o instrumento RST-PQ se destaca por apresentar quatro fatores para avaliar o fator BAS/SR e uma correlação adequada entre BIS e FFFS.

Conforme mencionado anteriormente, para ser considerado um instrumento adequado para a avaliação da rRST, o questionário deve possuir uma medida de SR/ BAS multifatorial e subescalas separadas para BIS e FFFS. Ainda, o pesquisador deve ter cuidado ao escolher medidas que propõem modelos fatoriais diferenciando Fight, Flight e Freeze, pois o ideal é que o sistema FFFS seja medido por meio de uma única escala (Corr, 2016). Por fim, para medir adequadamente o sistema BIS, é sugerido que sejam consideradas diferentes respostas de reação diante de conflito, incluindo itens que expressem traços de ansiedade e respostas de aproximação defensiva (e.g., cautela excessiva, receio ao tomar decisões).

Dentre os instrumentos desenvolvidos para avaliar a rRST, o RST-PQ (Corr \& Cooper, 2016) apresenta uma estrutura fatorial adequada ao modelo teórico e empírico do fator BAS (Gray \& McNaughton, 2000; Krupić et al., 2016). O RST-PQ também apresenta subescalas distintas para avaliar medo (FFFS) e ansiedade (BIS), com correlações moderadas entre as subescalas e com uma distinção entre medo e ansiedade de acordo com o proposto pela rRST. Uma característica adicional do instrumento foi a elaboração de uma subescala de Defensive Fight. Correlações entre medidas de luta defensiva e BAS vêm sendo descritas na teoria e altos índices de covariância são observados entre Defensive Fight e BAS (Corr \& Cooper, 2016). Isso ocorre uma vez que ambos os fatores podem ser considerados construtos relacionados mais às respostas de aproximação a estímulos do ambiente do que à evitação. Apesar disso, o RST-PQ ainda precisa ser melhor investigado à luz da rRST (Krupić et al., 2016). Diante do exposto, dentre os instrumentos apresentados nesta revisão, sugere-se que o mais adequado teórica e estatisticamente à rRST é o RST-PQ.

\section{MEDIDAS DE CONDICIONAMENTO}

Com o intuito de auxiliar o leitor a identificar respostas comportamentais atribuídas a cada sistema neuroanatômico da RST em tarefas de condicionamento, artigos com alguns dos principais procedimentos operantes utilizados para $\mathrm{o}$ teste de hipóteses da rRST sobre o reforçamento podem ser vistos na Tabela 2. As tarefas citadas são exemplos úteis para a mensuração da SR e SP. Procedimentos operantes que envolvem comportamento de escolha ou extinção de respostas previamente reforçadas foram selecionados por possibilitar o acesso a resultados relacionados a mais de um sistema neuroanatômico. Na Tabela 2, as linhas representam estudos independentes e as colunas BAS, BIS e FFFS indicam os resultados comportamentais e a relação destes com cada sistema de acordo com a rRST. 
Tabela 2

Exemplos de Procedimentos de Condicionamento Operante para o Teste de Hipóteses da rRST e Resultados Comportamentais Associadas aos Sistemas $B A S$, BIS e FFFS

\begin{tabular}{|c|c|c|c|c|c|c|c|}
\hline \multirow{2}{*}{ Autor (Ano) } & \multirow{2}{*}{ Amostra } & \multirow{2}{*}{$\begin{array}{c}\text { Procedimentos } \\
\text { Operantes }\end{array}$} & \multirow{2}{*}{ Escala } & \multicolumn{3}{|c|}{ Resultados e Relação com os Sistemas Neuroanatômicos } & \multirow{2}{*}{ HSC/HSS } \\
\hline & & & & BAS & BIS & FFFS & \\
\hline Aberg et al. (2016) & $\begin{array}{l}\text { Grupos por } \\
\text { desempenho: } \\
\text { reward } \\
\text { learners } \mathrm{e} \\
\text { avoidance } \\
\text { learners }\end{array}$ & $\begin{array}{l}\text { Probability } \\
\text { Selection Task }\end{array}$ & SPSRQ & $\begin{array}{l}\text { + Escolha } \\
\text { de respostas } \\
\text { previamente } \\
\text { reforçadas no grupo } \\
\text { reward learners }\end{array}$ & $\notin$ & $\begin{array}{c}\text { + Esquiva } \\
\text { de respostas } \\
\text { previamente } \\
\text { punidas no grupo } \\
\text { avoidance learners }\end{array}$ & HSS \\
\hline $\begin{array}{l}\text { Avila \& Parcet } \\
(2000)\end{array}$ & $\begin{array}{l}\text { Grupos: } \\
\mathrm{SR}+/ \mathrm{SP}+, \\
\mathrm{SR}+/ \mathrm{SP}- \\
\mathrm{SR}-/ \mathrm{SP}+ \\
\mathrm{SR}-/ \mathrm{SP}-\end{array}$ & $\begin{array}{l}\text { Choice Task/ } \\
\text { Extinction }\end{array}$ & SPSRQ & $\begin{array}{c}\text { + Respostas em } \\
\text { opções com reforço } \\
\text { contínuo e } \\
+ \text { Resistência à } \\
\text { extinção no grupo } \\
\text { SR+/SP- }\end{array}$ & $\begin{array}{c}+ \text { Omissão de } \\
\text { respostas durante } \\
\text { extinção no grupo } \\
\text { SR-/SP+ }\end{array}$ & $\notin$ & HSC \\
\hline $\begin{array}{l}\text { Corr \& Thompson } \\
\text { (2014) }\end{array}$ & $\begin{array}{l}\text { Amostra } \\
\text { Total }\end{array}$ & $\begin{array}{l}\text { Card } \\
\text { Perseveration } \\
\text { Task }\end{array}$ & BIS/BAS & $\begin{array}{l}\text { Correlação + entre } \\
\text { BAS autorrelatado e } \\
\text { escolha de respostas } \\
\text { previamente } \\
\text { reforçadas }\end{array}$ & $\begin{array}{c}\text { Correlação + entre } \\
\text { BIS autorrelatado } \\
\text { e tempo de reação } \\
\text { em milissegundos } \\
\text { diante de estímulos } \\
\text { que sinalizavam } \\
\text { punição }\end{array}$ & $\notin$ & HSS \\
\hline $\begin{array}{l}\text { Kambouroupoulos } \\
\& \text { Staiger (2004) }\end{array}$ & $\begin{array}{l}\text { Amostra } \\
\text { Total } \\
\text { e Grupos: } \\
\text { SR+/SP+, } \\
\text { SR+/SP--, } \\
\text { SR-/SP+, } \\
\text { SR-/SP- }\end{array}$ & $\begin{array}{l}\text { The Card } \\
\text { Arranging } \\
\text { Reward } \\
\text { Responsivity } \\
\text { Objective } \\
\text { Task/Q-Task }\end{array}$ & SPSRQ & $\begin{array}{l}\text { Correlação + entre } \\
\text { SR autorrelatado } \\
\text { e viés de respostas } \\
\text { em condições de } \\
\text { reforço positivo }\end{array}$ & $\begin{array}{c}\text { + Tempo de reação } \\
\text { em milissegundos } \\
\text { diante de estímulos } \\
\text { que sinalizavam } \\
\text { punição no grupo } \\
\text { SR+/SP+ }\end{array}$ & $\notin$ & HSC \\
\hline Smillie et al. (2007) & $\begin{array}{l}\text { Amostra } \\
\text { Total do } \\
\text { Estudo } 2\end{array}$ & $\begin{array}{l}\text { The } \\
\text { Categorization } \\
\text { Task }\end{array}$ & $\mathrm{BIS} / \mathrm{BAS}$ & $\begin{array}{c}\text { Correlação + entre } \\
\text { BAS autorrelatado } \\
\text { e escolha de } \\
\text { estímulos que } \\
\text { sinalizam reforço } \\
\text { positivo }\end{array}$ & $\begin{array}{l}\text { Correlação + entre } \\
\text { BIS autorrelatado } \\
\text { e omissão de } \\
\text { respostas diante } \\
\text { de estímulos que } \\
\text { sinalizam punição }\end{array}$ & $\notin$ & HSS \\
\hline
\end{tabular}

Nota. Instrumentos de autorrelato - SPSRQ: Sensitivity to Punishment and Sensitivity to Reward Questionnaire; BIS/BAS: Behavioral Inhibition System/ Behavioral Approach System. Personalidade - SR: Sensibilidade à Recompensa; SP: Sensibilidade à Punição. HSS: Hipótese dos Sistemas Separados; HSC: Hipótese dos Sistemas Conjuntos. $\notin$ indica que não há resultados comportamentais de acordo com o sistema da coluna; + Indica aumento ou relação positiva.

Na Tabela 2, é possível visualizar alguns dos principais resultados comportamentais relacionados à SR (BAS). No estudo de Aberg et al. (2016), os participantes foram agrupados pelo desempenho que apresentaram em uma tarefa de escolha, na qual a aprendizagem por reforçamento positivo e por reforçamento negativo (i.e., esquiva de escolhas previamente punidas) era avaliada. Participantes que apresentaram uma maior aprendizagem por reforçamento positivo, chamados de reward learners, também apresentaram escores maiores de SR. No estudo de Ávila e Parcet (2000), grupos de participantes com $\mathrm{SR}+/ \mathrm{SP}-$ demonstraram um viés maior de resposta para opções com reforços mais frequentes do que participantes $\mathrm{SR}+/ \mathrm{SP}+, \mathrm{SR}-/ \mathrm{SP}-\mathrm{e} \mathrm{SR}-/ \mathrm{SP}+$. O fator de impulsividade (Fun Seeking) do instrumento BIS/BAS foi correlacionado com uma tendência a comportamentos de risco sinalizada pela maior escolha de opções previamente reforçadas com pontos, independentemente da possibilidade de perder pontos (Corr \& Thompson, 2014). No estudo de Kambouroupoulos e Staiger (2004), escores de SR medidos por autorrelato também foram correlacionados com um maior número de respostas quando participantes eram reforçados positivamente em uma tarefa de agrupamento de cartões. Por fim, a SR avaliada por autorrelato (subescala BAS) também foi correlacionada com uma maior frequência de respostas em um experimento no qual os participantes recebiam reforço positivo para $80 \%$ das respostas corretas em uma tarefa de tomada de decisão (Smillie et al., 2007).

Em indivíduos $\mathrm{SR}+/ \mathrm{SP}-$, também é possível observar subprodutos do reforçamento, como a dificuldade em modificar respostas previamente reforçadas quando o reforço positivo não está mais ocorrendo, um efeito chamado de resistência à extinção (Ávila \& Parcet, 2000). Tal resultado só foi encontrado quando os participante do estudo foram classificados conforme os escores de SR e SP a partir da mediana das escalas, formando quatro grupos $(\mathrm{SR}+/ \mathrm{SP}+$, 
$\mathrm{SR}+/ \mathrm{SP}-, \mathrm{SR}-/ \mathrm{SP}+, \mathrm{SR}-/ \mathrm{SP}-)$, o que possibilitou uma comparação entre grupos de acordo com a HSC (Corr, 2004).

A dissecação do traço SP em BIS e FFFS aumenta a abrangência da rRST ao mesmo tempo em que dificulta a experimentação. A lentificação durante situações de conflito é um dos principais marcadores da aproximação defensiva do BIS. Tarefas com tomada de decisão, extinção e mudança na característica motivacional de estímulos (e.g., de reforçadores para punidores) são desejáveis para avaliar o funcionamento desse sistema de SP (Leue \& Beauducel, 2008). Dois resultados podem ser destacados na Tabela 2 : atrasos no tempo de reação e erros de omissão. Atrasos ao responder são um marcador de conflito por sinalizar a inibição do comportamento e início da aproximação defensiva (Leue \& Beauducel, 2008). No estudo de Corr e Thompson (2014), o atraso em uma tarefa de tomada de decisão, indicada pelo tempo de reação entre tentativas, apresentou uma correlação positiva com o BIS autorrelatado. Já os erros de omissão em tarefas de reforçamento com conflito indicam a ocorrência de esquiva passiva de estímulos aversivos (Leue \& Beauducel, 2008). A omissão de respostas durante procedimentos de extinção ou logo após punição foi observada em grupos de participantes com maiores níveis de SP, um resultado que está relacionada ao BIS (Ávila \& Parcet, 2000; Kambouroupoulos \& Staiger, 2004; Smillie et al., 2007).

O sistema FFFS de SP, por outro lado, deve reagir ativamente diante de estímulos aversivos condicionados e incondicionados, facilitando respostas de esquiva (McNaughton \& Corr, 2008). No experimento de Aberg et al. (2016), indivíduos que evitaram com mais frequência respostas previamente punidas em uma tarefa de escolha demonstraram um escore maior de SP. Respostas de fuga de estímulos aversivos, chamadas também de esquiva ativa, são características do FFFS e indicam aprendizagem por reforçamento negativo (McNaughton \& Corr, 2008).

\section{Recomendações para a escolha de medidas de condicionamento operante}

Duas características devem ser ponderadas na elaboração e escolha de tarefas de condicionamento: a característica dos estímulos e o tipo de tarefa de aprendizagem medida (Ávila \& Torrubia, 2008). A literatura da RST prevê que tarefas operantes com reforço positivo são, necessariamente, apetitivas por produzirem a seleção de respostas por suas consequências e por gerar subprodutos do reforçamento positivo, como a aproximação do estímulo antecedente correlacionado ao reforço (Ávila \& Torrubia, 2008). Tal concepção supõe uma relação hedônica entre esses estímulos antecedentes e o próprio reforço, sendo o termo recompensa utilizado com frequência para se referir ao reforço positivo na RST (Gray \& McNaughton, 2000). De maneira oposta, a RST assume que processos de extinção e punição positiva são considerados aversivos e que um estímulo correlacionado a consequências aversivas adquire parte de suas propriedades.

Um ponto a ser considerado no planejamento de testes da relação entre personalidade e comportamento suposta pela rRST refere-se à escolha dos estímulos reforçadores e punidores. Estímulos condicionados são predominantemente utilizados, tais como pontuações ou feedbacks verbais (e.g., "bom" ou "ruim"; Leue \& Beauducel, 2008). Leue \& Beauducel sugerem que, na ausência de estímulos reforçadores ou punidores incondicionados em procedimentos operantes com humanos, o melhor estímulo condicionado para ser utilizado como consequência reforçadora seria o dinheiro. Entretanto, esses autores apontam que antecipar o valor de uma recompensa ou punição hipotética por meio de um estímulo discriminativo que sinalize a magnitude da consequência (e.g., ganhar ou perder 200 pontos) pode ter efeitos semelhantes.

Um segundo ponto a ser considerado no teste de relação entre personalidade e condicionamento da rRST é o tipo de procedimento operante utilizado e as medidas comportamentais escolhidas. A partir de uma metanálise sobre a relação dos traços de personalidade da RST com a aprendizagem por condicionamento operante, Leue e Beauducel (2008) fornecem algumas recomendações para a elaboração e seleção de procedimentos operantes e medidas: (a) procedimentos que envolvam comportamento de escolha ou que incluam extinção são úteis para avaliar respostas mediadas pelo BIS por produzirem reações de conflito; (b) procedimentos operantes que possibilitam estabilizar o responder antes de iniciar o procedimento de extinção ou antes de adicionar uma punição de respostas previamente reforçadas possuem mais chances de aumentar o tamanho de efeito estatístico quando grupos contrastantes (e.g., SP+ e SP-) são comparados; (c) em procedimentos operantes, medidas como erros de omissão e tempo de reação deveriam ser usadas para isolar os efeitos do BIS.

Finalmente, ao escolher a medida de condicionamento operante, o pesquisador deve considerar tanto o efeito isolado de SR e SP (HSS) quanto o efeito conjunto dos traços de personalidade (HSC). Corr (2004) aponta que, em tarefas de condicionamento nas quais estímulos altamente apetitivos ou aversivos (e.g., dinheiro e choque) são apresentados separadamente para cada resposta, é provável que ocorra um resultado mais de acordo com a HSS, dado que os efeitos dos sistemas de SR (BAS) e SP (BIS e FFFS) sobre a aprendizagem serão mais facilmente isolados. No entanto, quando a mesma resposta operante pode produzir estímulos apetitivos ou aversivos ou quando os reforçadores e punidores são de baixa magnitude (e.g., pontos), um resultado de acordo com a HSC é esperado, dado que efeitos conjuntos dos sistemas podem facilitar a aprendizagem em indivíduos SR+/SP- ou SR-/SP+ (Corr, 2004). 


\section{CONSIDERAÇÕES FINAIS}

No presente artigo, o desenvolvimento da RST e suas principais hipóteses foram descritas com o objetivo de apresentar ao leitor um modelo psicobiológico de personalidade que pressupõe relação entre personalidade e condicionamento. Somente nos últimos 10 anos, ao menos cinco novos instrumentos foram propostos para avaliar as dimensões de personalidade da rRST (Čolović et al., 2018; Corr \& Cooper, 2016; Jackson, 2009; Reuter et al., 2015; Smederevac et al., 2014). No entanto, ainda é habitual o uso de instrumentos de autorrelato derivados da versão antiga da teoria (e.g., BIS/BAS e SPSRQ; Aberg et al., 2016; Corr \& Thompson, 2014). A avaliação precisa dos sistemas BIS, BAS e FFFS por meio de autorrelato ainda é um desafio.

A rRST possui revisões teóricas e metanálises com diversas evidências dos efeitos da SR e da SP sobre o condicionamento (Ávila \& Torrubia, 2008; Corr, 2004; Leue $\&$ Beauducel, 2008), validando o pressuposto central da teoria. Todavia, nem sempre os procedimentos de condicionamento utilizados são úteis para diferenciar respostas de esquiva (FFFS) daquelas de aproximação defensiva (BIS), uma distinção importante na rRST (Leue \& Beuaducel, 2008). Ainda, Ávila e Torrubia (2008) argumentam que o controle de variáveis estranhas como o sono e o nível de estresse durante os experimentos é mais limitado no estudo com seres humanos. Os autores vão além e argumentam também que o controle de variáveis cognitivas, como a expectativa dos participantes durante as tarefas de condicionamento, pode ser útil, já que não se sabe o quanto elas interferem na relação entre personalidade e condicionamento.
As recomendações aqui contidas são úteis para pesquisadores e profissionais da saúde mental interessados em um modelo psicobiológico de personalidade amplamente estudado pelas neurociências, psiquiatria e psicologia experimental. No entanto, este artigo possui limitações. Uma revisão sistemática não foi realizada devido ao fato de que os autores gostariam de apresentar a teoria de maneira abrangente, incluindo as principais hipóteses e medidas. Explicações sobre a ação fisiológica dos sistemas BAS, BIS e FFFS não foram abordadas neste artigo. No entanto, a literatura sobre as bases neurobiológicas da RST/rRST é vasta (Gray \& McNaughton, 2000; McNaughton \& Corr, 2008; Reuter et al., 2015; Whisman et al., 2011). Por fim, dados que mostram associações diferenciais entre os fatores de personalidade da RST e a presença de transtornos psiquiátricos não foram abordadas ao longo do texto (ver Bijttebier et al., 2009 para uma revisão).

É relevante destacar que toda a bibliografia utilizada na revisão está em inglês e artigos em português sobre as hipóteses da RST/rRST e suas medidas não foram encontrados, o que destaca a importância do presente estudo, o qual apresenta não só a teoria, mas também suas principais hipóteses, procedimentos e medidas em língua portuguesa. Os diversos procedimentos e medidas expostos nesta revisão, bem como as bases teóricas da rRST, fomentam a relação entre personalidade e psicologia experimental e fornecem um modelo teórico para diferenças individuais na aprendizagem que pode ser útil para o estudo do comportamento humano.

\section{REFERÊNCIAS}

Aberg, K. C., Doell, K. C., \& Schwartz, S. (2016). Linking Individual Learning Styles to Approach-Avoidance Motivational Traits and Computational Aspects of Reinforcement Learning. PLoS One, 11(11), e0166675. https://doi.org/10.1371/journal. pone. 0166675

Ávila, C., \& Parcet, M. A. (2000). The Role of Gray's Impulsivity in Anxiety-Mediated Differences in Resistance to Extinction. European Journal of Personality, 14(3), 185-198. https:// doi.org/10.1002/1099-0984(200005/06)14:3\%3C185::AIDPER370\%3E3.0.CO;2-U

Ávila, C., \& Torrubia, R. (2008). Performance and Conditioning Studies. Em P. Corr (Org.), The Reinforcement Sensitivity Theory of Personality (pp. 228-260). Cambridge University Press. https://doi.org/10.1017/CBO9780511819384

Benjamin, J., Li, L., Patterson, C., Greenberg, B. D., Murphy, D. L., \& Hamer, D. H. (1996). Population and Familial Association between the D4 Dopamine Receptor Gene and Measures of Novelty Seeking. Nature Genetics, 12(1), 81-84. https://doi. org/10.1038/ng0196-81

Bijttebier, P., Beck, I., Claes, L., \& Vandereycken, W. (2009). Gray's Reinforcement Sensitivity Theory as a Framework for Research on Personality-Psychopathology Associations. Clinical Psychology Review, 29(5), 421-430. https://doi. org/10.1016/j.cpr.2009.04.002
Carver, C. S., \& White, T. L. (1994). Behavioral Inhibition, Behavioral Activation, and Affective Responses to Impending Reward and Punishment: The BIS/BAS Scales. Journal of Personality and Social Psychology, 67(2), 319-333. http:// dx.doi.org/10.1037/0022-3514.67.2.319

Čolović, P., Smederevac, S., Oljača, M., Nikolašević, Ž., \& Mitrović, D. (2018). A Short Measure of the Revised Reinforcement Sensitivity Theory-RSQ17. The Journal of Psychology, 152(3), 133-150. https://doi.org/10.1080/00223 980.2017.1419158

Conner, B. T., Rahm-Knigge, R. L., \& Jenkins, A. L. (2018). Revision and Clarification of the Sensitivity to Punishment Sensitivity to Reward Questionnaire. Personality and Individual Differences, 121, 31-40. https://doi.org/10.1016/j. paid.2017.09.016

Corr, P. J. (2004). Reinforcement Sensitivity Theory and Personality. Neuroscience \& Biobehavioral Reviews, 28(3), 317-332. https://doi.org/10.1016/j.neubiorev.2004.01.005

Corr, P. J. (2016). Reinforcement Sensitivity Theory of Personality Questionnaires: Structural Survey with Recommendations. Personality and Individual Differences, 89, 60-64. https://doi. org/10.1016/j.paid.2015.09.045

Corr, P. J., \& Cooper, A. J. (2016). The Reinforcement Sensitivity Theory of Personality Questionnaire (RST-PQ): Development 
and Validation. Psychological Assessment, 28(11), 1427-1440. http://psycnet.apa.org/doi/10.1037/pas0000273

Corr, P. J., \& Thompson, S. J. (2014). Pause for Thought: Response Perseveration and Personality in Gambling. Journal of Gambling Studies, 30(4), 889-900. https://doi.org/10.1007/ s10899-013-9395-4

Eysenck, H. (1979). The Conditioning Model of Neurosis. Behavioral and Brain Sciences, 2(2), 155-166. https://doi. org/10.1017/S0140525X00061653

Eysenck H.J., \& Levey A. (1972). Conditioning, IntroversionExtraversion and the Strength of the Nervous System. Em V. D. Nebylitsyn, \& J. A. Gray (Orgs.), Biological Bases of Individual Behavior (pp. 206-220). Academic Press.

Gray, J. A. (1982). The Neuropsychology of Anxiety: An Enquiry into the Septo-Hippocampal System. Oxford University Press.

Gray, J. A., \& McNaughton, N. (2000). The Neuropsychology of Anxiety: An Enquiry into the Septo-Hippocampal System (2a $E d$.). Oxford University Press. https://doi.org/10.1093/acprof :oso/9780198522713.001.0001

Jackson, C. J. (2009). Jackson-5 Scales of Revised Reinforcement Sensitivity Theory (r-RST) and Their Application to Dysfunctional Real-World Outcomes. Journal of Research in Personality, 43(4), 556-569. https://doi.org/10.1016/j. jrp.2009.02.007

Kambouropoulos, N., \& Staiger, P. K. (2004). Personality and Responses to Appetitive and Aversive Stimuli: The Joint Influence of Behavioural Approach and Behavioural Inhibition Systems. Personality and Individual Differences, 37(6), 11531165. https://doi.org/10.1016/j.paid.2003.11.019

Krupić, D., Corr, P. J., Ručević, S., Križanić, V., \& Gračanin, A. (2016). Five Reinforcement Sensitivity Theory (RST) of Personality Questionnaires: Comparison, Validity and Generalization. Personality and Individual Differences, 97, 19-24. https://doi.org/10.1016/j.paid.2016.03.012

Leue, A., \& Beauducel, A. (2008). A Meta-Analysis of Reinforcement Sensitivity Theory: On Performance Parameters in Reinforcement Tasks. Personality and Social Psychology Review, 12(4), 353-369. https://doi. org/10.1177\%2F1088868308316891

Markett, S., Montag, C., \& Reuter, M. (2014). In Favor of Behavior: On the Importance of Experimental Paradigms in Testing Predictions from Gray's Revised Reinforcement Sensitivity Theory. Frontiers in Systems Neuroscience, 8(184), 1-3. https:// doi.org/10.3389/fnsys.2014.00184

McNaughton, N., \& Corr, P. J. (2008). The Neuropsychology of Fear and Anxiety: A Foundation for Reinforcement Sensitivity Theory. Em P. Corr (Org.), The Reinforcement Sensitivity
Theory of Personality (pp. 44-94). Cambridge University Press. https://psycnet.apa.org/doi/10.1017/CBO9780511819384.003

Mobbs, D., Petrovic, P., Marchant, J. L., Hassabis, D., Weiskopf, N., Seymour, B., Dolan, R. J., \& Frith, C. D. (2007). When Fear is Near: Threat Imminence Elicits Prefrontal-Periaqueductal Gray Shifts in Humans. Science, 317(5841), 1079-1083. https://doi. org/10.1126/science. 1144298

Pickering, A., \& Corr, P. J. (2008). J. A. Gray's Reinforcement Sensitivity Theory (RST) of Personality. Em G. J. Boyle, G. Matthews, \& D. H. Saklofske (Orgs.), The Sage Handbook of Personality Theory and Assessment (pp. 239-256). Sage.

Reuter, M., Cooper, A. J., Smillie, L. D., Markett, S., \& Montag, C. (2015). A New Measure for the Revised Reinforcement Sensitivity Theory: Psychometric Criteria and Genetic Validation. Frontiers in Systems Neuroscience, 9(38), 1-12. https://doi.org/10.3389/fnsys.2015.00038

Reuter, M., Schmitz, A., Corr, P. J., \& Hennig, J. (2006). Molecular Genetics Support Gray's Personality Theory: The Interaction of COMT and DRD2 Polymorphisms Predicts the Behavioral Approach System. International Journal of Neuropsychopharmacology, 9(2), 155-166. https://doi. org/10.1017/S1461145705005419

Smederevac, S., Mitrović, D., Čolović, P., \& Nikolašević, Ž. (2014). Validation of the Measure of Revised Reinforcement Sensitivity Theory Constructs. Journal of Individual Differences, 35, 1221. https://doi.org/10.1027/1614-0001/a000121

Smillie, L. D., Dalgleish, L. I., \& Jackson, C. J. (2007). Distinguishing between Learning and Motivation in Behavioral Tests of the Reinforcement Sensitivity Theory of Personality. Personality and Social Psychology Bulletin, 33(4), 476-489. https://doi.org/10.1177\%2F0146167206296951

Torrubia, R., Avila, C., Moltó, J., \& Caseras, X. (2001). The Sensitivity to Punishment and Sensitivity to Reward Questionnaire (SPSRQ) as a Measure of Gray's Anxiety and Impulsivity Dimensions. Personality and Individual Differences, 31(6), 837-862. https://doi.org/10.1016/S01918869(00)00183-5

Walker, B. R., Jackson, C. J., \& Frost, R. (2017). A Comparison of Revised Reinforcement Sensitivity Theory with Other Contemporary Personality Models. Personality and Individual Differences, 109, 232-236. https://doi.org/10.1016/j. paid.2016.12.053

Whisman, M. A., Richardson, E. D., \& Smolen, A. (2011). Behavioral Inhibition and Triallelic Genotyping of the Serotonin Transporter Promoter (5-HTTLPR) polymorphism. Journal of Research in Personality, 45(6), 706-709. https://doi. org/10.1016/j.jrp.2011.08.009 